BMJ Open Diabetes

Research $\&$ Care

\title{
Adverse differences in cardiometabolic risk factor levels between individuals with pre-diabetes and normal glucose metabolism are more pronounced in women than in men: the Maastricht Study
}

Rianneke de Ritter (D , , ${ }^{1,2}$ Simone J S Sep, ${ }^{1,2,3}$ Carla J H van der Kallen, ${ }^{1,2}$ Miranda T Schram (D) , 1,2,4 Annemarie Koster, ${ }^{5,6}$ Abraham A Kroon, ${ }^{1,2}$ Marleen M J van Greevenbroek (1) ,1,2 Simone J P M Eussen, 2,7 Pieter C Dagnelie, ${ }^{1,2}$ Marit de Jong (D) , ${ }^{8}$ Rimke C Vos (D) , ${ }^{8,9}$ Mark Woodward, ${ }^{10,11,12}$ Michiel L Bots, ${ }^{8}$ Sanne A E Peters (D) , ${ }^{8,10}$ Coen D A Stehouwer ${ }^{1,2}$

\section{ABSTRACT}

Objective To investigate whether adverse differences in levels of cardiovascular risk factors in women than men, already established when comparing individuals with and without diabetes, are also present before type 2 diabetes onset.

Research design and methods In a population-based cohort study of individuals aged $40-75$ years $(n=3410$; $49 \%$ women, $29 \%$ type 2 diabetes (oversampled by design)), we estimated associations with cardiometabolic and lifestyle risk factors of (1) pre-diabetes and type 2 diabetes (reference category: normal glucose metabolism) and (2) among non-diabetic individuals, of continuous levels of hemoglobin A1c ( $\mathrm{HbA1c})$. Age-adjusted sex differences were analyzed using linear and logistic regression models with sex interaction terms.

Results In pre-diabetes, adverse differences in cardiometabolic risk factors were greater in women than men for systolic blood pressure (difference, $3.02 \mathrm{~mm} \mathrm{Hg}$; $95 \%$ Cl:-0.26 to 6.30), high-density lipoprotein (HDL) cholesterol (difference, $-0.10 \mathrm{mmol} / \mathrm{L} ; 95 \% \mathrm{Cl}:-0.18$ to -0.02 ), total-to-HDL cholesterol ratio (difference, 0.22; $95 \% \mathrm{Cl}:-0.01$ to 0.44 ), triglycerides (ratio: $1.11 ; 95 \%$ Cl: 1.01 to 1.22$)$, and inflammation markers Z-score (ratio: 1.18; $95 \% \mathrm{Cl}: 0.98$ to 1.41). In type 2 diabetes, these sex differences were similar in direction, and of greater magnitude. Additionally, HbA1c among nondiabetic individuals was more strongly associated with several cardiometabolic risk factors in women than men: per one per cent point increase, systolic blood pressure (difference, $3.58 \mathrm{~mm} \mathrm{Hg}$; $95 \% \mathrm{Cl}$ : -0.03 to 7.19 ), diastolic blood pressure (difference, $2.10 \mathrm{~mm} \mathrm{Hg} ; 95 \% \mathrm{Cl}:-0.02$ to 4.23 ), HDL cholesterol (difference, $-0.09 \mathrm{mmol} / \mathrm{L} ; 95 \%$ Cl: -0.19 to 0.00 ), and low-density lipoprotein cholesterol (difference, $0.26 \mathrm{mmol} / \mathrm{L} ; 95 \% \mathrm{Cl}: 0.05$ to 0.47 ). With regard to lifestyle risk factors, no consistent pattern was observed.

\section{Significance of this study}

What is already known about this subject?

- There is compelling evidence that type 2 diabetes is a stronger risk factor for cardiovascular disease (CVD) in women than in men.

- Previous studies have shown that adverse differences in cardiovascular risk factors between individuals with type 2 diabetes and normal glucose metabolism are more pronounced in women than in men However, it remains uncertain whether sex differences in cardiovascular risk factor levels already emerge before the development of type 2 diabetes.

What are the new findings?

- Our results are consistent with the concept that the more adverse changes in cardiometabolic risk factors in women than men already emerge, as a continuous process, before the development of type 2 diabetes.

How might these results change the focus of research or clinical practice?

> Our results suggest that early cardiovascular risk management in women, before the development of diabetes, might help to reduce the sex difference in cardiovascular complications of diabetes.

Conclusion Our results are consistent with the concept that the more adverse changes in cardiometabolic risk factors in women (than men) arise as a continuous process before the onset of type 2 diabetes.

\section{INTRODUCTION}

There is compelling evidence that type 2 diabetes is a stronger risk factor for 
cardiovascular disease (CVD), mainly of macrovascular origin, in women than in men. ${ }^{1-3}$ Several studies have shown that adverse differences in cardiovascular risk factors between individuals with type 2 diabetes and normal glucose metabolism (NGM) are more pronounced in women than in men. ${ }^{4-7}$ However, whether sex differences in cardiovascular risk factor levels already emerge before the development of type 2 diabetes remains uncertain. Nevertheless, such evidence could help to further understand the biological processes underlying the observed excess diabetes-associated risk of CVD in women.

More detailed research is required to identify whether adverse differences in levels of cardiometabolic and lifestyle risk factors between individuals with pre-diabetes and NGM are already more pronounced in women than in men, and if so, how these differences relate to those observed in type 2 diabetes. Furthermore, deeper insights are needed in the course of emergence of sex differences in various risk factors from NGM to the onset of type 2 diabetes. A larger deterioration in cardiometabolic risk factors, before the onset of type 2 diabetes, might induce a more pronounced exposure to a hazardous cardiometabolic environment in women than in men. ${ }^{1}$ This larger deterioration in risk factor levels might be driven by sex differences in body anthropometry and differential patterns of fat storage ${ }^{58}$ In addition to cardiometabolic risk factors, sex differences in patterns of lifestyle have also been associated with type 2 diabetes ${ }^{9-15}$ and might contribute to women's excess risk on diabetes vascular complications as well.

Therefore, we investigated, in a large cohort study with detailed information on risk factors, sex differences in the associations with cardiometabolic and lifestyle risk factors of (1) pre-diabetes and type 2 diabetes (reference category: NGM) and (2) among individuals without type 2 diabetes, of continuous levels of hemoglobin A1c (HbA1c).

\section{RESEARCH DESIGN AND METHODS \\ Study design and population}

Data were used from the Maastricht Study, an observational prospective population-based cohort study. The rationale and methodology have been described previously. ${ }^{16}$ In brief, the Maastricht Study focuses on the etiology, pathophysiology, complications, and comorbidities of type 2 diabetes and is characterized by an extensive phenotyping approach. Individuals aged between 40 and 75 years at study baseline, and living in the southern part of the Netherlands, were eligible to participate. Participants were recruited through mass media campaigns, and from the municipal registries and the regional Diabetes Patient Registry via mailings. Recruitment was stratified according to known type 2 diabetes status, with an oversampling of individuals with type 2 diabetes, for reasons of efficiency. The present report includes cross-sectional data from the first 3451 participants, who completed the baseline survey between November 2010 and September 2013. Participants with other types of diabetes than type 2 diabetes or with a history of pancreatectomy were excluded $(n=41)$. The examinations of each participant were performed within a time window of 3 months. All participants gave written informed consent.

\section{Glucose metabolism status}

To determine glucose metabolism status (GMS), all participants underwent a standardized 2-hour 75 gram OGTT after fasting overnight. For safety reasons, participants using insulin or with a fasting glucose level above $11.0 \mathrm{mmol} / \mathrm{L}$, as determined by a finger prick, did not undergo the OGTT. For these individuals $(n=64)$, fasting glucose level and information about diabetes medication were used to determine GMS. GMS was defined according to the ${ }^{17}$ criteria into NGM, impaired fasting glucose, impaired glucose tolerance (combined as prediabetes), and type 2 diabetes. ${ }^{17}$ Participants on blood glucose lowering medication were classified as having type 2 diabetes.

\section{Cardiometabolic risk factors}

Waist and hip circumference, body mass index (BMI), fasting serum levels of triglycerides, total cholesterol, high-density lipoprotein (HDL) cholesterol and lowdensity lipoprotein (LDL) cholesterol (calculated by the Friedewald equation, or measured by direct assay if total cholesterol $<1.3 \mathrm{mmol} / \mathrm{L}$ or triglycerides $>4.5 \mathrm{mmol} / \mathrm{L}$ ), fasting plasma levels of glucose and insulin, HbAlc, markers of inflammation, systolic and diastolic office blood pressure, and 24 hours ambulatory systolic and diastolic blood pressure were determined as described elsewhere. $^{16}$

Insulin sensitivity was estimated by HOMA2 (homeostasis model assessment), and was expressed as a percentage of a normal reference population. ${ }^{18}$ Markers of inflammation include C-reactive protein (CRP), serum amyloid A (SAA), soluble intercellular adhesion molecule-1 (sICAM-1), interleukin-6 (IL-6), IL-8, and tumor necrosis factor alpha (TNF $\alpha)$. Markers of inflammation were measured only once; therefore, the possible association with type 2 diabetes could be underestimated. To address this concern and to summarize the available data, an inflammation sum score has been constructed. ${ }^{19}$ First, the values of each inflammation marker were logtransformed, since residuals were skewed. Next, these values were expressed as a Z-score, that is, (value in the individual minus the mean value in the study population) divided by the standard deviation. The inflammation marker Z-score was subsequently calculated as (Z-score of CRP +Z-score of SAA +Z-score of sICAM-1 +Z-score of IL-6 + Z-score of IL-8 +Z-score of TNF $\alpha$ ) / 6 , and standardized.

\section{Lifestyle risk factors}

Physical activity level, smoking status, alcohol use, and diet were determined as described elsewhere. ${ }^{16}$ Physical activity was measured by use of a tri-axial accelerometer 
for 7 days (ActivPal, PAL Technologies, Glasgow, UK). ${ }^{20}$ Smoking status was categorized into never, former, and current smoker; alcohol consumption was categorized into non-consumers, low consumers ( $\leq 7$ glasses per week for women and $\leq 14$ glasses per week for men), and high consumers ( $>7$ glasses per week for women and $>14$ glasses per week for men). ${ }^{16}$ Adherence to the Greek Mediterranean diet score was calculated from a validated food frequency questionnaire. ${ }^{21}$ The Mediterranean diet score can take a value from zero to nine points, which indicates adherence to consumption of presumed beneficial components. ${ }^{22}$ The diet score was categorized into low adherence (0-3 points), medium adherence (4,5 points), and high adherence (6-9 points).

\section{Covariates and population characteristics}

A questionnaire was used to assess postmenopausal status in women, history of CVD, physical activity (hours of moderate to vigorous physical activity per week), and level of education. ${ }^{16}$ Level of education was categorized into low, medium, and high. Glucose-lowering, lipidmodifying and antihypertensive medication, as well as postmenopausal hormone replacement therapy, was assessed during a medication interview where generic name, dose, and frequency were registered. ${ }^{16}$

\section{Statistical analyses}

Statistical analyses were performed using SPSS V.25.0 for Windows (IBM SPSS, IBM Corp, Armonk, NY, USA). Population characteristics were described as mean $\pm \mathrm{SD}$, median (IQR), or n (\%), as appropriate. Variables were log-transformed if residuals were skewed.

Linear and logistic regression analyses were performed to estimate the associations of (1) pre-diabetes and type 2 diabetes (reference category: NGM) and (2) HbAlc (continuously) among individuals without type 2 diabetes with cardiovascular risk factors after adjusting for age. To test for sex differences, interaction terms (eg, dummy-coded GMS variables * sex or HbA1c* sex) were incorporated into the regression models. $\mathrm{A}_{\text {interaction }}<0.10$ was considered statistically significant, as commonly used for statistical interaction testing. ${ }^{23}$ Since the main goal of this study was to test for sex differences, both $p$ values $<0.05$ and $<0.10$ are shown and results are presented with a $95 \%$ CI. In addition, an interaction term of age by sex was incorporated into the regression models to correct for potential sex-specific effects of age as a covariate. Finally, sex-stratified analyses were performed to estimate the sex-specific associations between GMS and HbAlc with cardiovascular risk factors. Since missing data differ by dependent variable, a separate complete case analysis was performed for each individual dependent variable. No imputation of missing values was performed.

Several additional analyses were performed. First, sex differences in the associations of GMS with additional anthropometric risk factors and separate markers of inflammation were determined. Second, sensitivity analyses were performed to estimate the associations of GMS with 24 hours ambulatory blood pressure (instead of office blood pressure), since 24 hours ambulatory blood pressure is the gold standard. For statistical efficiency, because more data on 24 hours ambulatory blood pressure were missing, office blood pressure was primarily used.

Third, in separate analyses, associations of GMS with cardiovascular risk factors were additionally adjusted for BMI, duration of diabetes, smoking status, alcohol consumption, physical activity, educational level, and antihypertensive and lipid-modifying medication use, where appropriate. For medication use, an interaction term of medication use by sex was incorporated into the regression models to correct for observed sex-specific effects of medication.

Finally, additional analyses of the sex differences in the age-adjusted associations of GMS with cardiovascular risk factors were conducted among individuals without prevalent CVD (analysis population $\mathrm{n}=2753$ ), and excluding premenopausal women (analysis population $\mathrm{n}=3017$ ). Strata sizes of individuals with prevalent CVD, and of premenopausal women only, were too small to perform subgroup analyses of sex differences (premenopausal women: NGM $n=262$, pre-diabetes $n=38$, type 2 diabetes $\mathrm{n}=38$; history of CVD in women and men, respectively: NGM $n=128$ and $n=94$, pre-diabetes $n=28$ and $n=42$, type 2 diabetes $\mathrm{n}=63$ and $\mathrm{n}=197$ ).

\section{RESULTS}

\section{Characteristics of the study population}

The study population consisted of 3410 individuals, 1654 women (age 58.7 78.2 years) and 1756 men (age 60.9 \pm 8.1 years). Of these, 1924 ( $57 \%$ women) had NGM, 511 ( $46 \%$ women) had pre-diabetes and 975 ( $32 \%$ women) had type 2 diabetes (table 1 ).

In general, women with NGM had a more favorable cardiovascular risk profile than men with NGM (table 1). For both women and men, the cardiometabolic risk profile deteriorated with GMS (table 1, figure 1). Women and men with pre-diabetes and type 2 diabetes had a higher BMI, waist and hip circumference, blood pressure and markers of inflammation, a more adverse lipid profile and lower insulin sensitivity than individuals of the same sex with NGM. Both women and men with pre-diabetes and type 2 diabetes, on average, were less physically active, were more likely to smoke, had an unhealthier diet, yet consumed less alcohol than individuals of the same sex with NGM.

Sex differences in the associations of pre-diabetes and type 2 diabetes with cardiometabolic and lifestyle risk factors

Adverse differences in cardiometabolic risk factors between individuals with pre-diabetes and NGM were more pronounced in women than in men for office systolic blood pressure (SBP; beta for interaction: $3.02 \mathrm{~mm} \mathrm{Hg}$; $95 \%$ CI: -0.26 to 6.30$)$, HDL cholesterol $(-0.10 \mathrm{mmol} / \mathrm{L}$; $95 \%$ CI: -0.18 to -0.02$)$, total-to-HDL cholesterol ratio 


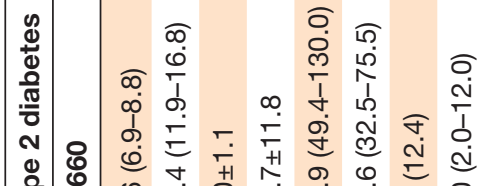

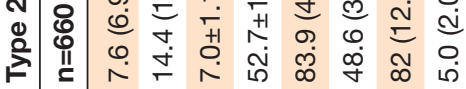

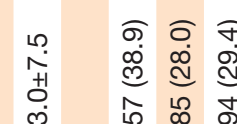

๓

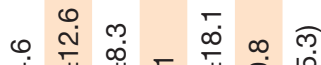

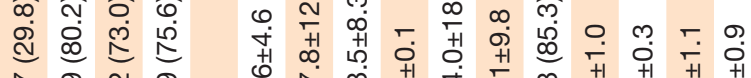

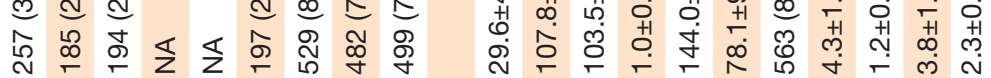

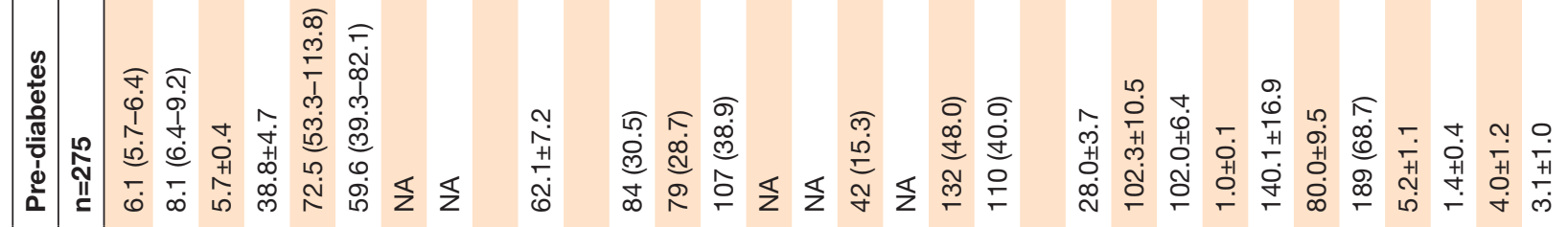

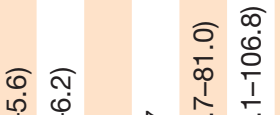

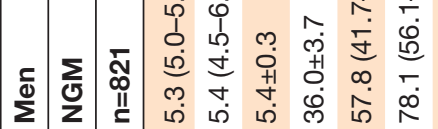

$+\sqrt{2} \leqslant$

₹ $\widehat{\infty} \widehat{0}$

ก)

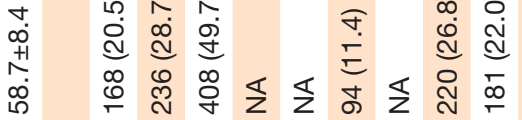

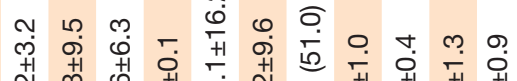

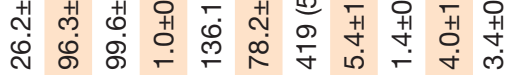

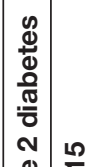

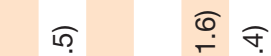

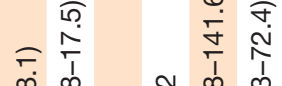

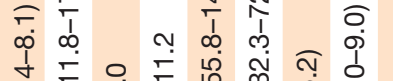

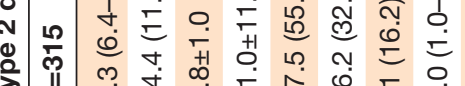

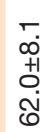

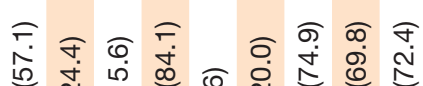

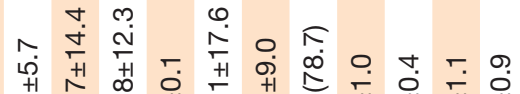

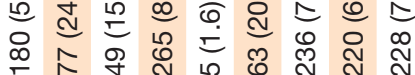

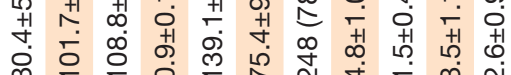

10 边

ल

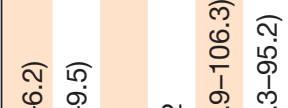

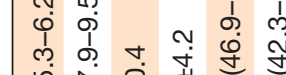

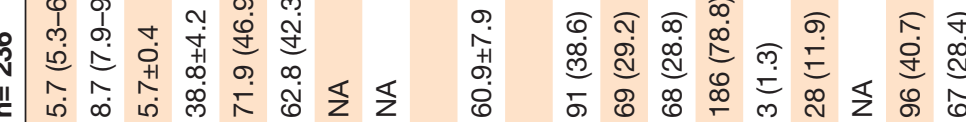

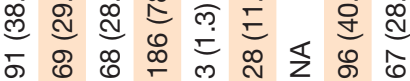

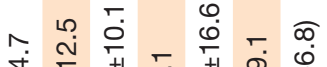

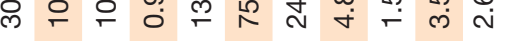

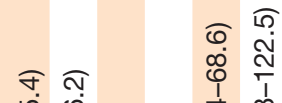

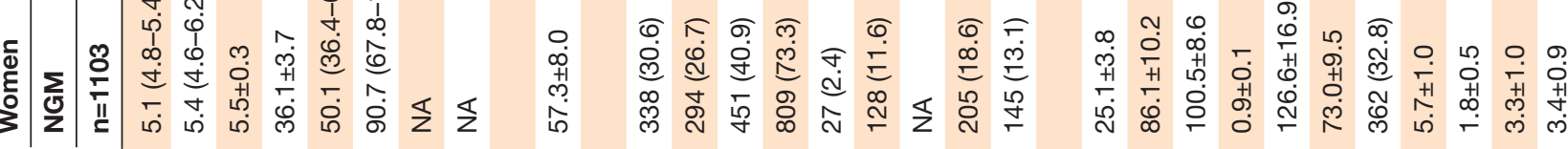
a

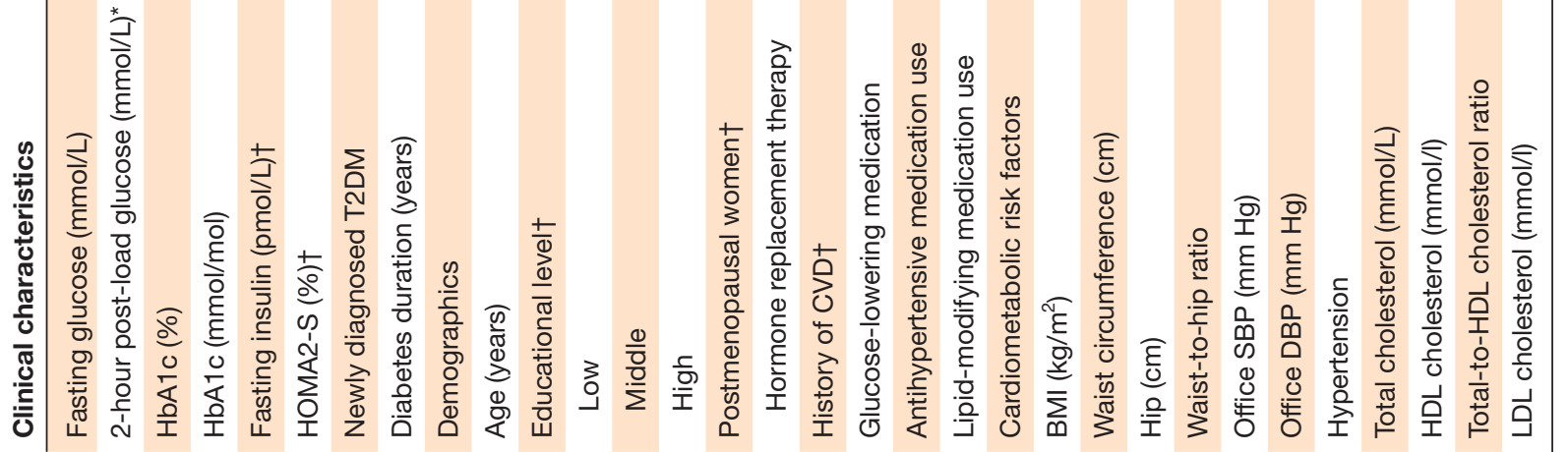




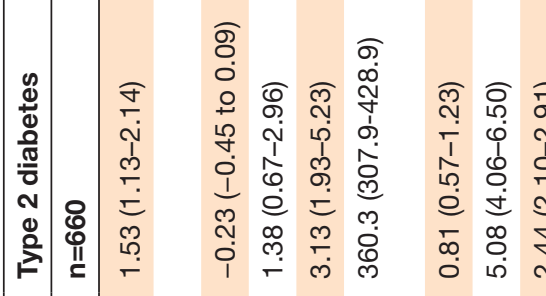

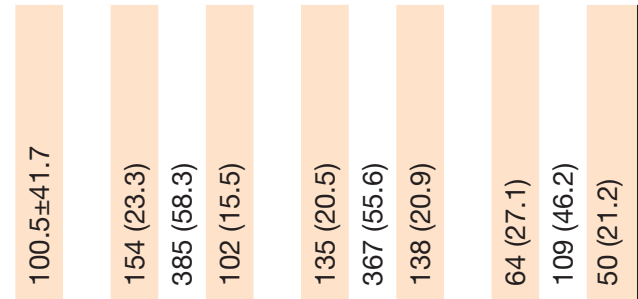

क्ल

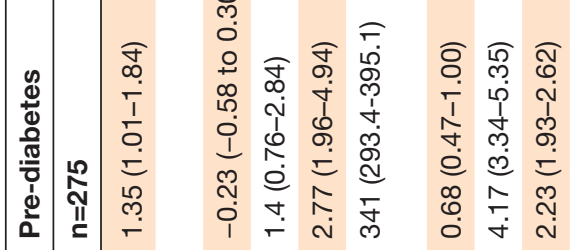

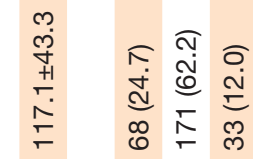

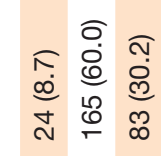

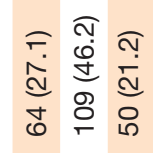

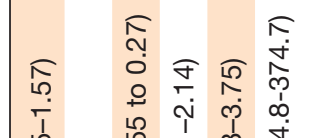

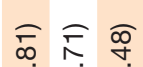

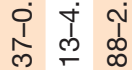

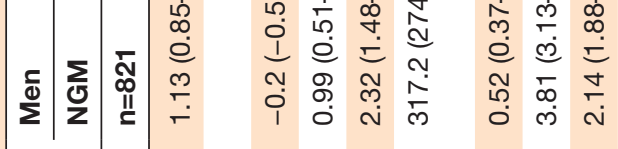

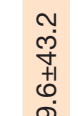

ลุ สุ

赵审

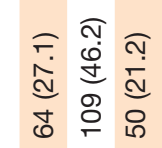

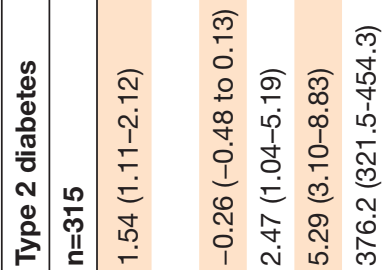

등

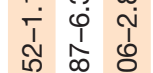

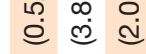

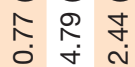

$\infty$
$\infty$
$\stackrel{\infty}{1}$
$\stackrel{1}{0}$
$\stackrel{0}{0}$

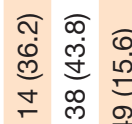

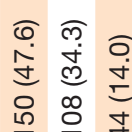

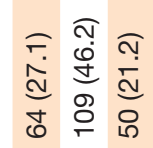

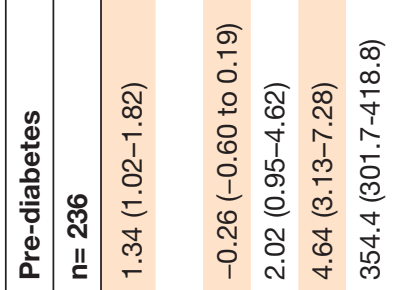

వ

i.

7.

ชั

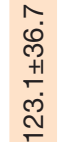

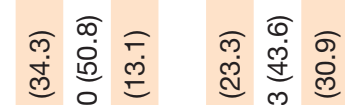

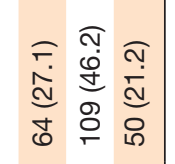

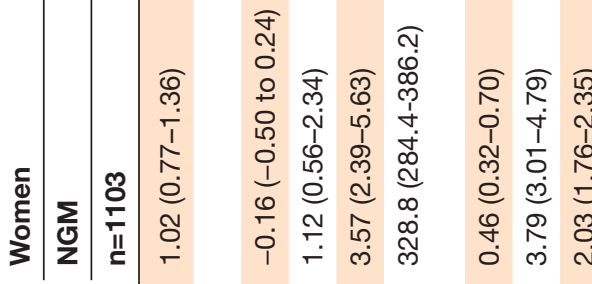

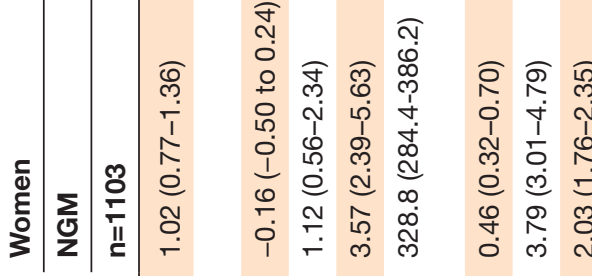

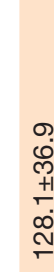

क

员 웅 웃

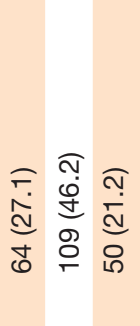

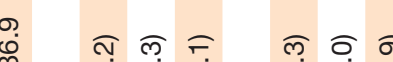

त.

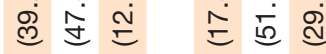

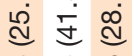

学 䜣

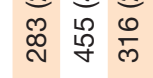

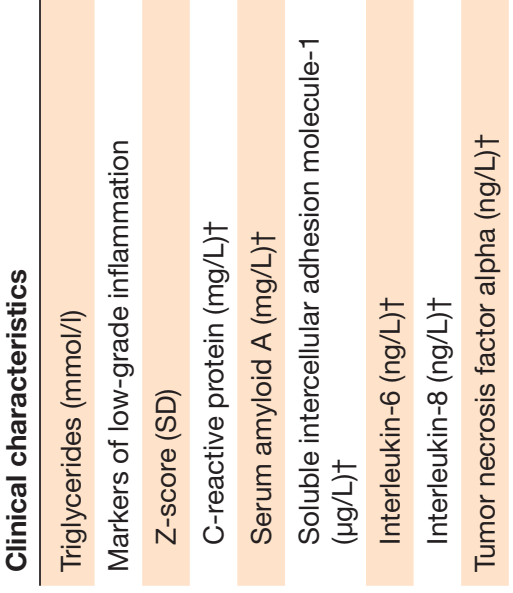

䑻

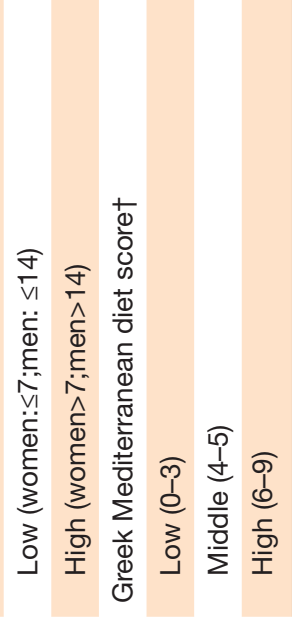

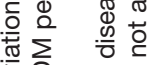

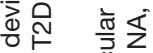

离

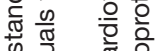

+1

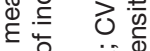


A

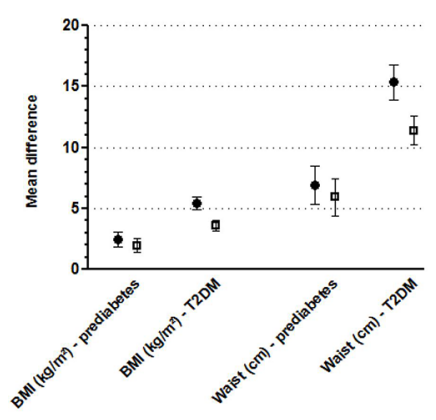

B

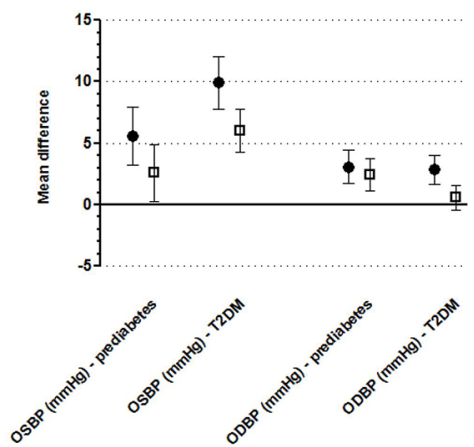

c

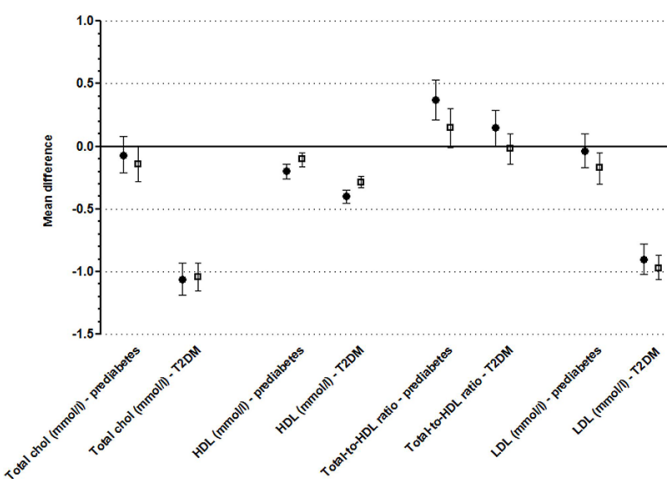

D

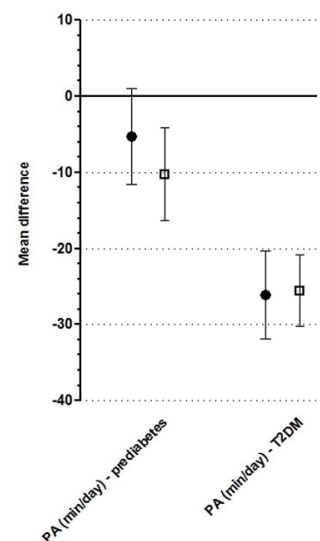

E

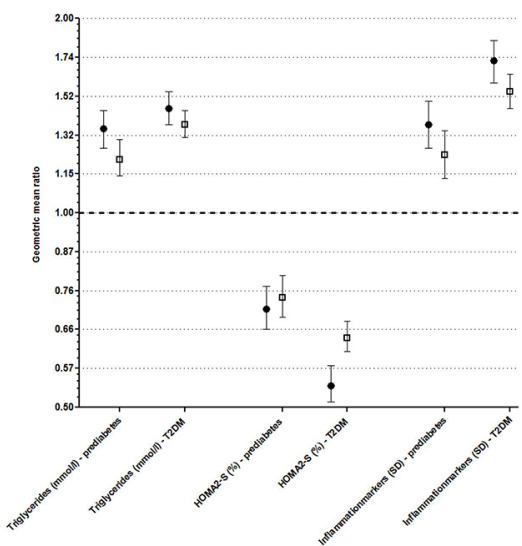

F

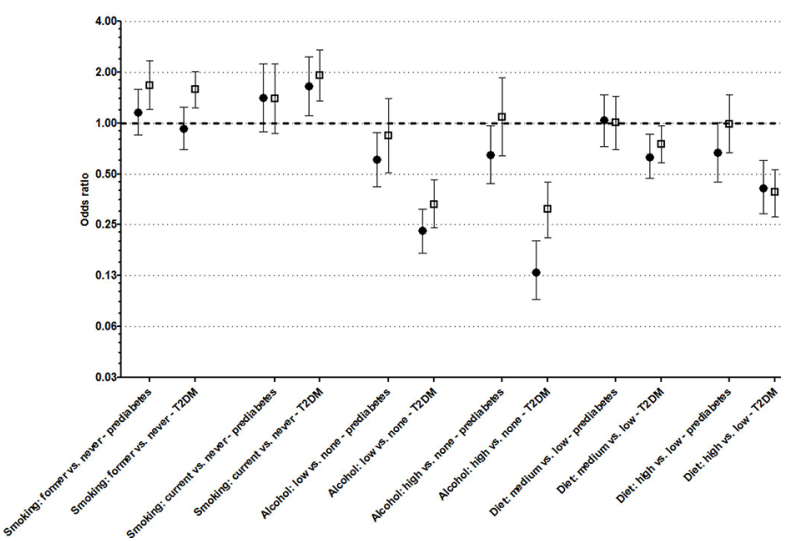

Figure 1 Age-adjusted sex-specific mean differences of levels of cardiometabolic and lifestyle risk factors between (pre) diabetes and normal glucose metabolism as references category. Results are expressed as age-adjusted linear regression coefficients, which indicate mean differences in the levels of BMI and waist circumference, office blood pressure, lipid levels, and physical activity, geometric mean ratios in the levels of triglycerides, insulin sensitivity and markers of inflammation, and odds ratios in lifestyle risk factors. Circles represent comparisons within women and squares represent comparisons within men. Black lines represent 95\% Cls. T2DM, type 2 diabetes mellitus; OSBP, office systolic blood pressure, ODBP, office diastolic blood pressure; Total chol, total cholesterol; PA, physical activity - mean number of stepping minutes perday; HOMA2-S, Homeostasis Model Assessment of insulin sensitivity.

(0.22; $95 \%$ CI: -0.01 to 0.44$)$, triglycerides $(1.11 ; 95 \%$ CI: 1.01 to 1.22 ; times higher geometric mean ratio) and markers of inflammation Z-score (1.18 SD; 95\% CI: 0.98 to 1.41 ) (table 2 , figure $1 \mathrm{~B}, \mathrm{C}$ and $\mathrm{E}$ ). These sex differences in pre-diabetes versus NGM were similar to, although smaller than, those observed in type 2 diabetes. No statistically significant sex differences were observed in the associations of pre-diabetes with BMI, waist and hip circumference, HOMA-2 and office diastolic blood pressure (DBP), whereas in the association with type 2 diabetes, differences in these risk factors were relatively more adverse in women than in men (table 2, online supplementary table 2, figure $1 \mathrm{~A}, \mathrm{~B}$ and $\mathrm{E}$ ). With regard to lifestyle risk factors, no consistent pattern was observed (table 2, figure $1 \mathrm{D}$ and $\mathrm{F}$ ).

\section{Additional analyses}

With regard to anthropometric risk factors, results of hip circumference were generally consistent with the results from waist circumference and BMI in the main analysis (online supplementary table 1 ). With regard to individual inflammation markers, results were generally consistent with the main analysis, except for IL-8 (online supplementary table 1). In contrast to findings for office blood pressure, no stronger association in women than men between pre-diabetes and type 2 diabetes with 24 hours ambulatory blood pressure was observed (online supplementary table 1 ).

After additional adjustment for BMI, sex differences in the associations of pre-diabetes and type 2 diabetes with cardiometabolic risk factors were attenuated. Some statistically significant sex differences disappeared, notably the associations between type 2 diabetes and total-to-HDL cholesterol, and between both pre-diabetes and type 2 diabetes and markers of inflammation (Z-score) (online supplementary table 2).

After additional adjustment for duration of diabetes, sex differences in the associations of type 2 diabetes with cardiometabolic and lifestyle risk factors did not materially change (online supplementary table 3 ).

After additional adjustment for the use of antihypertensive medication, sex differences in the associations of pre-diabetes and type 2 diabetes with office blood pressure were attenuated or disappeared. Adjustment for lipid-modifying medication only minimally changed the 


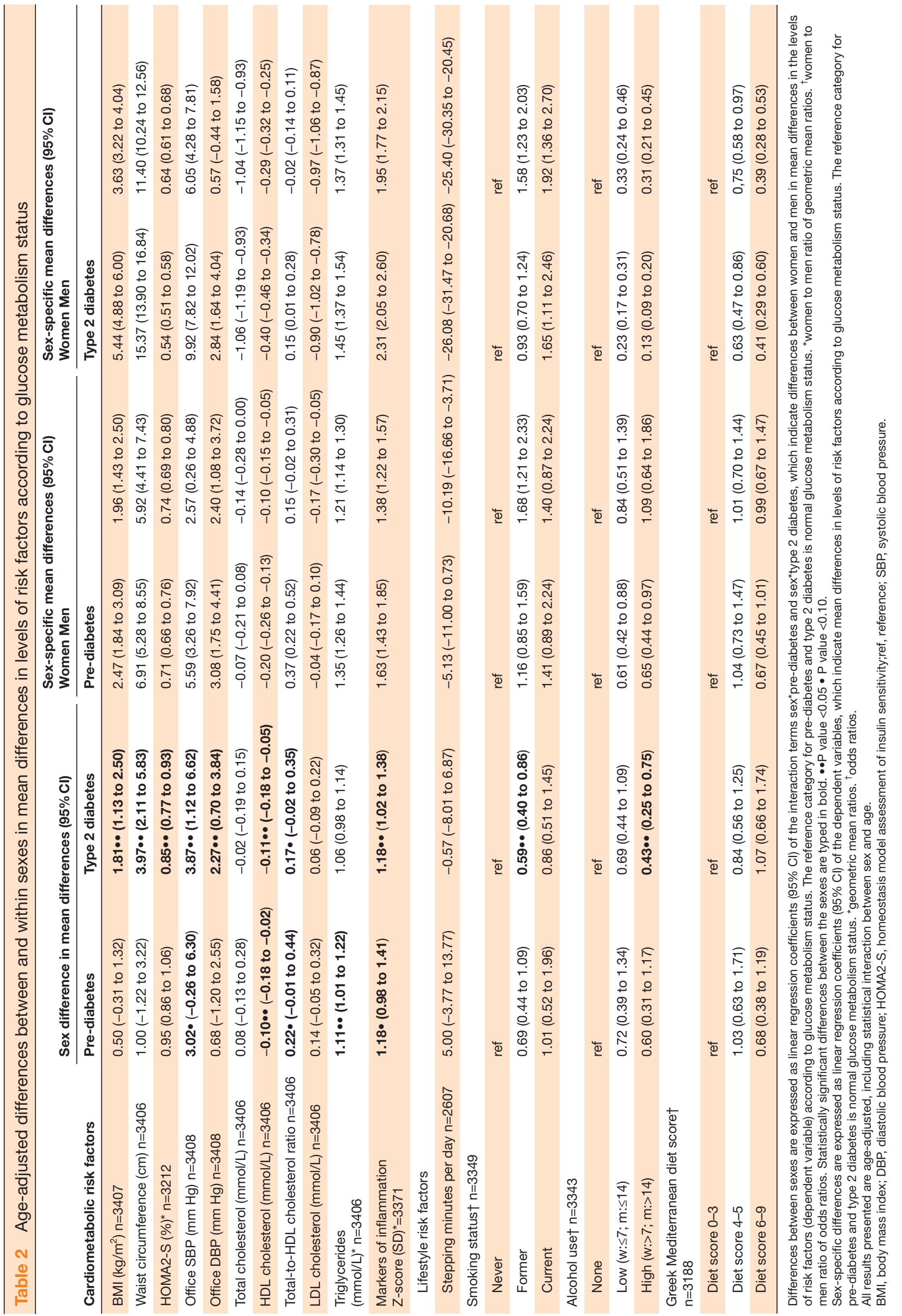


observed sex differences in lipid levels (online supplementary table 4).

Adjustment for lifestyle factors and educational level, and exclusion of participants with prevalent CVD only minimally changed the sex differences observed. Exclusion of premenopausal women attenuated the observed sex differences in blood pressure, lipid profile, and markers of inflammation (online supplementary table 5).

\section{Sex differences in the continuous association of HbA1c with cardiometabolic and lifestyle risk factors among individuals without type 2 diabetes}

HbA1c was more strongly associated with several cardiometabolic risk factors in women than men. Sex differences to the disadvantage of women, per one percent point increase in HbAlc, were observed for office SBP (3.58 mm Hg; 95\% CI: -0.03 to 7.19), office DBP $(2.10 \mathrm{~mm} \mathrm{Hg} ;-0.02$ to 4.23$)$, HDL cholesterol $(-0.09 \mathrm{mmol} / \mathrm{L} ; 95 \%$ CI: -0.19 to 0.00$)$, and LDL cholesterol $(0.26 \mathrm{mmol} / \mathrm{L} ; 95 \%$ CI: 0.05;0.47) (figure 2, online supplementary table 6 ). With regard to lifestyle risk factors, no consistent pattern was observed (online supplementary table 6 ).

\section{CONCLUSIONS}

In this study, we determined sex differences in the associations of both GMS (pre-diabetes and type 2 diabetes) and of continuous measures of HbAlc, with traditional cardiometabolic risk factors, inflammatory profile, and lifestyle risk factors for CVD. We demonstrated that adverse differences in cardiometabolic risk factors between individuals with pre-diabetes and NGM are already more pronounced in women than in men. These sex differences were similar to, but smaller than, the sex differences observed in type 2 diabetes. In addition, HbAlc in individuals without type 2 diabetes is more strongly associated with several cardiometabolic risk factors in women than in men. Taken together, these observations are consistent with the concept that these sex differences reflect a continuous process that already emerges in early dysglycemia.

To our knowledge, sex differences associated with oral glucose tolerance test (OGTT) based pre-diabetes and continuous $\mathrm{HbAlc}$ among individuals without type 2 diabetes have not been reported previously. However, longitudinal studies did focus on sex differences related to the conversion to diabetes or dysglycemia. ${ }^{24-26}$ These studies found that women who eventually developed diabetes experienced a greater deterioration in fasting plasma glucose, lipid levels, and BMI during their nondiabetic state. Furthermore, women had a relatively greater endothelial dysfunction, more hypertension and a greater degree of dysregulated fibrinolysis and coagulation than their male counterparts. ${ }^{24-26}$

Type 2 diabetes has been shown to be associated with adverse differences in cardiovascular risk factor levels, which in line with our findings, are more pronounced in women than in men. ${ }^{4-7} 26$ Correspondingly, BMI and waist circumference differ more between women with and without diabetes than between men with and without diabetes. ${ }^{726}$ When newly diagnosed with type 2 diabetes, the BMI of women has shown to be almost $2 \mathrm{~kg} / \mathrm{m}^{2}$ higher despite similar levels of HbA1c. ${ }^{27}{ }^{28}$ These sex differences suggest a link with a differential pattern of fat storage and insulin resistance between women and men. ${ }^{58}$ It is hypothesized that women need to attain higher levels of BMI and deteriorate related risk factors to a greater extent than men to develop insulin resistance and eventually diabetes. ${ }^{29}{ }^{30}$ Although not statistically significant, we found a tendency toward more adverse differences in levels of BMI and insulin sensitivity in women than in men associated with pre-diabetes, and with HbAlc in those without type 2 diabetes. Furthermore, after adjustment for BMI, all significantly different sex differences in cardiometabolic risk factors associated with pre-diabetes and type 2 diabetes were attenuated. Thus, even small sex differences in BMI may induce a greater deterioration of related risk factors before the onset of type 2 diabetes in women than men.

Women seem to have a greater reserve capacity regarding glucose metabolism impairment, ${ }^{31}$ with extended exposure to the pre-diabetic state. On average, men have pre-diabetes for 8.5 years and women for 10.3 years before they develop type 2 diabetes, ${ }^{32}$ which may also expand the duration of exposure to cardiometabolic hazards in women. ${ }^{31}$ Since no consistent pattern was observed in sex differences in the associations of pre-diabetes and type 2 diabetes, and among individuals without type 2 diabetes, of levels of HbAlc with lifestyle risk factors, biological factors are more likely to underlie the relatively higher diabetes-associated cardiovascular risks in women. Further research into biological factors, specifically into fat distribution and metabolism, is needed to unravel the underlying mechanisms of sex differences in the association between type 2 diabetes and vascular function.

The sex differences that we identified were not attributable to duration of diabetes, pharmacological management, lifestyle, or educational level, except for the observed sex differences in blood pressure, which were absent or attenuated after adjustment for the use of antihypertensive medication. Notably, the observed sex differences in office and 24 hours ambulatory blood pressure were inconsistent (ie, present in office blood pressure only). This may indicate a stronger diabetesassociated white coat effect in women. In general, a higher prevalence of white coat hypertension in women has been repeatedly reported. ${ }^{33}$ The clinical relevance of white coat hypertension should not be underestimated as it has been associated with an increased risk of complications (eg, retinopathy and nephropathy). ${ }^{34}$

The exclusion of premenopausal women generally attenuated the observed sex differences, which could be explained by age and/or hormonal factors. ${ }^{10}$ The excess risk of vascular mortality among women associated with 


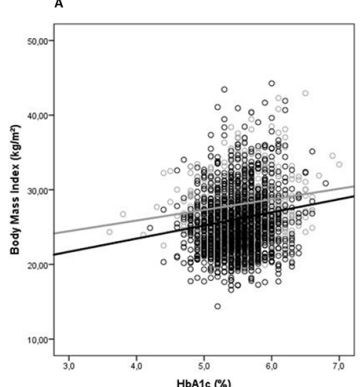

Beta $(95 \%-C l)$ Women: $1.76(1.17: 2.35)$
Beta (95\%-Cl) Men: $1.42(0.83 ; 2.02)$

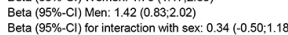

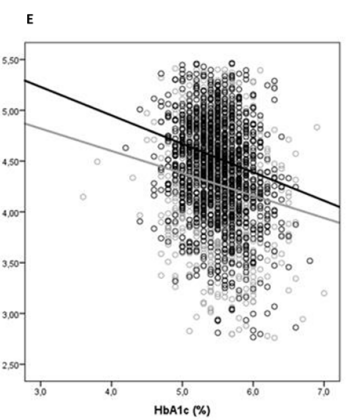

Beta (95\%-CI) Women *: $0.76(0.70 ; 0.82)$

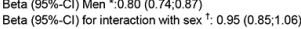

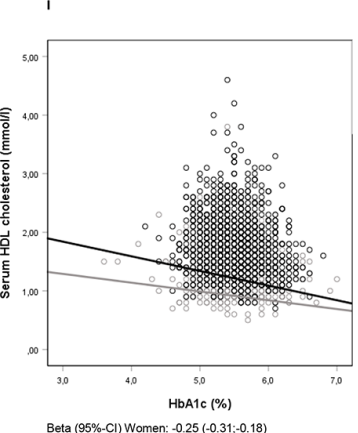
Betat $(95 \%-C)$ Women: $-0.25(-0.31 ;-0.18)$
Beta $(95 \%-C)$ Men: $0.15(-0.22-0.09)$

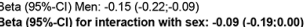

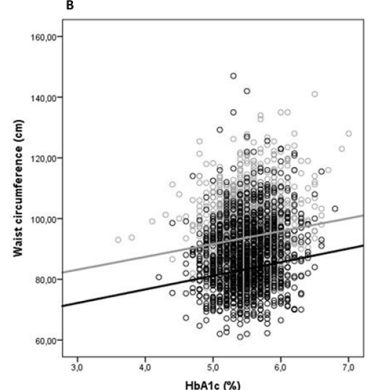

Beta (95\%-CI) Women: 4.48 (2.84:6.11) Beta (95\%-Cl) Men: $4.22(2.57: 5.58)$
Beta (95\%)-Cl) for interaction with sex: 0.25 -
$(-207.257)$

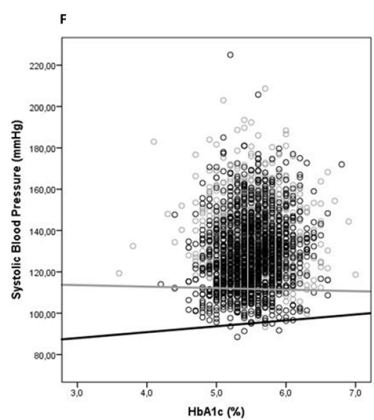

Beta (95\%-CI) Women: 2.85 (0.31:5.5.3\%
Beta (95\%-C) Men: $-0.73(-331: 1.14)$ Beta (95\%)-CI) for interaction with sex: 3.58
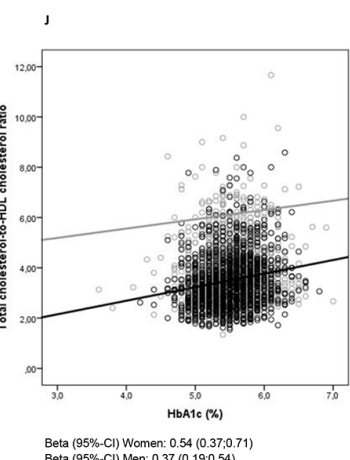
Beta (95\%-Cl) Women: $0.54(0.37 ; 0.71)$
Beta (95\%-Cl) Men: $0.37(0.19 ; 0.54)$
Beta (95\%)-Cl) for interaction with sex: 0.17
$(-0.07: 0.42)$

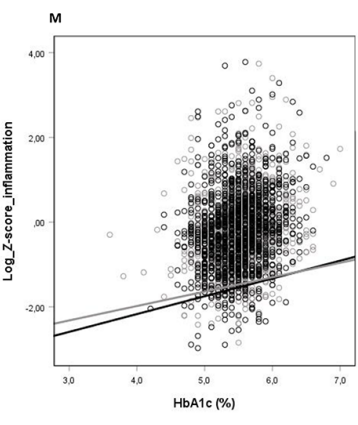
Beta (95\%-Cl) Women *: $1.51(1.32: 1.74)$
Beta (95\%-Cl) Men * : $1.40(1.22 ; 1.61)$

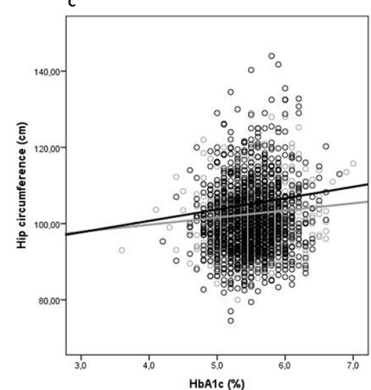

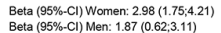

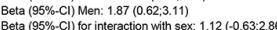

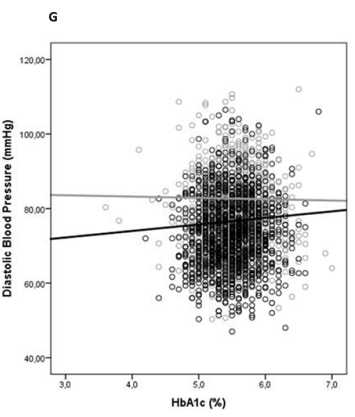

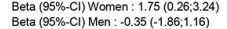

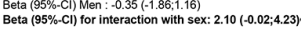

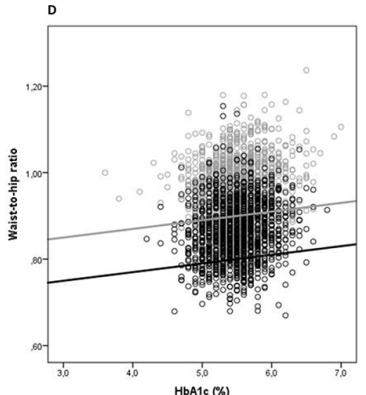

Beta (95\%-Cl) Wamen: 0.02 (0.017:0.03) Beta $(95 \%$-Cl) Men: 0.02 ( $0.01 ; 0.03)$
Beta $(95 \%$-Cl) for interaction vith sex 0.00 $(-0.02: 0.01)$

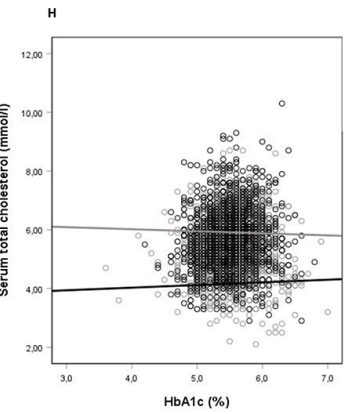

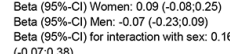

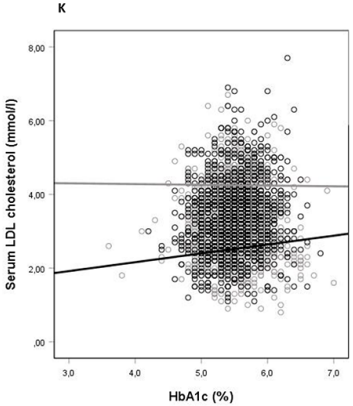

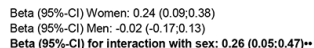

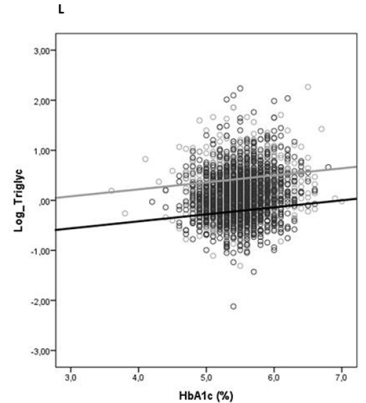

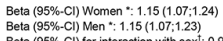
Bets

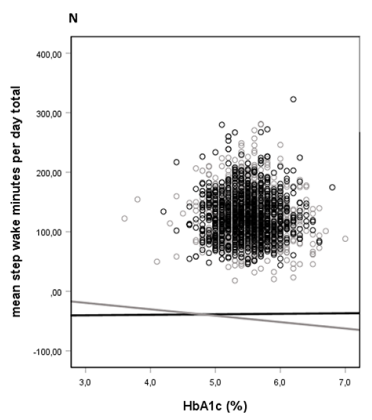

Betat (95\%)-CI) Women $0.02(-6.10 .7 .73)$
Beta

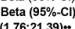

Figure 2 Sex-specific associations (black circles: women, gray circles: men) of HbA1c with body mass index (A), waist circumference $(B)$, hip circumference (C), waist-hip-ratio (D), HOMA2-S\% (E), office systolic blood pressure (F), office diastolic blood pressure $(\mathrm{G})$, total cholesterol $(\mathrm{H}), \mathrm{HDL}$ cholesterol (I), total-to-HDL cholesterol (J), LDL cholesterol (K), triglycerides (L), markers of inflammation Z-score (M), mean number of stepping minutes per day $(\mathrm{N})$ in participants without type 2 diabetes. Regression coefficients (beta) indicate the age-adjusted mean difference $(95 \% \mathrm{Cl})$ in cardiovascular risk factor levels per $1 \%$ point increase in $\mathrm{HbA} 1 \mathrm{c}$ for women (black lines) and men (gray lines). ${ }^{*}$ geometric mean ratios. ${ }^{\dagger}$ women to men ratio of geometric mean ratios. Mean differences that were statistically significantly different between the sexes are typed in bold. $\bullet p<0.05 \bullet p<0.10$. HbA1c, hemoglobin A1c; HDL, high-density lipoprotein; HOMA2-S, homeostasis model assessment of insulin sensitivity; LDL, low-density lipoprotein. 
diabetes is especially high among younger women (aged 35-59 years). ${ }^{3}$ Notably, we observed that the associations of age with blood pressure and lipid profile were dissimilar in women and men (sex*age interactions were incorporated in our regression models; data not shown). Future studies with larger sample sizes should focus on age-specific diabetes-associated sex differences in cardiovascular risk (factors) (eg, three-way interaction).

Strengths of our study include its population-based design combined with oversampling of individuals with type 2 diabetes, which enables an accurate comparison of individuals with and without type 2 diabetes. Second, this study benefits from its sample size, the detailed assessment of GMS by use of an OGTT, and the extensive phenotypic characteristics in all participants. The overall good glycemic control of individuals with type 2 diabetes in our population may have been the result of selecting relatively healthy individuals with type 2 diabetes, which could have led to certain associations being underestimated. However, we do not expect this to have affected men and women differently. The study also has some limitations. First, the data were cross-sectional; therefore, we cannot exclude reverse causality. Second, the percentage of women in the type 2 diabetes study population was about $10 \%$ point lower than that in the source population. ${ }^{35}$ If the apparent under-representation of women with type 2 diabetes was due to health selection, then the sex differences could be underestimated. At any rate, the recruitment strategy was the same for women as for men. ${ }^{16}$

In conclusion, the findings of this study demonstrate that the adverse differences in cardiometabolic risk factors between individuals with pre-diabetes and NGM are more pronounced in women than in men. Moreover, our results are consistent with the concept that these sex differences arise in a continuous process that starts long before the onset of type 2 diabetes. Sex differences in cardiometabolic risk factors have been proposed as an explanation for the higher relative risk of CVD associated with diabetes in women. Possibly, the higher relative risk of CVD in women is already present before the onset of type 2 diabetes, since we observed that sex differences in cardiometabolic risk factors are already existent in early dysglycemia. Tailoring cardiovascular risk management for women could benefit from this insight.

\section{Author affiliations}

${ }^{1}$ Department of Internal Medicine, Maastricht University Medical Centre+, Maastricht, The Netherlands

${ }^{2}$ CARIM Cardiovascular Research Institute Maastricht, Maastricht University, Maastricht, The Netherlands

${ }^{3}$ Adelante, Centre of Expertise in Rehabilitation and Audiology, Hoensbroek, The Netherlands

${ }^{4}$ Heart and Vascular Centre, Maastricht University Medical Centre+, Maastricht, The Netherlands

${ }^{5}$ Department of Social Medicine, Maastricht University, Maastricht, The Netherlands ${ }^{6}$ CAPHRI Care and Public Health Research Institute, Maastricht University,

Maastricht, The Netherlands

${ }^{7}$ Department of Epidemiology, Maastricht University, Maastricht, The Netherlands
${ }^{8}$ Julius Center for Health Sciences and Primary Care, University Medical Center Utrecht, Utrecht, The Netherlands

${ }^{9}$ Department of Public Health and Primary Care / LUMC-Campus, Leiden University Medical Center, The Hague, The Netherlands

${ }^{10}$ The George Institute for Global Health, University of Oxford, Oxford, United Kingdom

${ }^{11}$ The George Institute for Global Health, University of New South Wales, Sydney, New South Wales, Australia

${ }^{12}$ Department of Epidemiology, Johns Hopkins University, Baltimore, Maryland, USA

Contributors RdR wrote this paper, under supervision of SJSS, CJHvdK, SAEP, and CDAS. All authors reviewed the draft paper and provided critical intellectual content. All authors approved the final version of the manuscript and its submission to BMJ Open Diabetes Research \& Care. CDAS is taking responsibility for the contents of the article.

Funding This study was supported by ZonMw (project no 849200001), the European Regional Development Fund via OP-Zuid, the Province of Limburg, the Dutch Ministry of Economic Affairs (grant 310.041), Stichting De Weijerhorst (Maastricht, the Netherlands), the Pearl String Initiative Diabetes (Amsterdam, the Netherlands), CARIM, School for Cardiovascular Diseases (Maastricht, the Netherlands), School CAPHRI, Care and Public Health Research Institute (Maastricht, the Netherlands), NUTRIM, School of Nutrition and Translational Research in Metabolism (Maastricht, the Netherlands), Stichting Annadal (Maastricht, the Netherlands), Health Foundation Limburg (Maastricht, the Netherlands) and by unrestricted grants from Janssen-Cilag B.V. (Tilburg, the Netherlands), Novo Nordisk Farma B.V. (Alphen aan den Rijn, the Netherlands) and Sanofi-Aventis Netherlands B.V. (Gouda, the Netherlands).

Competing interests None declared.

Patient consent for publication Not required.

Ethics approval The study has been approved by the institutional medical ethical committee (NL31329.068.10) and the Minister of Health, Welfare and Sports of the Netherlands (Permit 131 088-1 05234 PG).

Provenance and peer review Not commissioned; externally peer reviewed.

Data availability statement Data are available upon reasonable request.

Open access This is an open access article distributed in accordance with the Creative Commons Attribution Non Commercial (CC BY-NC 4.0) license, which permits others to distribute, remix, adapt, build upon this work non-commercially, and license their derivative works on different terms, provided the original work is properly cited, appropriate credit is given, any changes made indicated, and the use is non-commercial. See: http://creativecommons.org/licenses/by-nc/4.0/.

\section{ORCID iDs}

Rianneke de Ritter http://orcid.org/0000-0003-2858-1874

Miranda T Schram http://orcid.org/0000-0001-8113-7604

Marleen M J van Greevenbroek http://orcid.org/0000-0002-2989-1631

Marit de Jong http://orcid.org/0000-0001-9260-4458

Rimke C Vos http://orcid.org/0000-0003-1074-6255

Sanne A E Peters http://orcid.org/0000-0003-0346-5412

\section{REFERENCES}

1 Peters SAE, Huxley RR, Woodward M. Diabetes as risk factor for incident coronary heart disease in women compared with men: a systematic review and meta-analysis of 64 cohorts including 858,507 individuals and 28,203 coronary events. Diabetologia 2014;57:1542-51.

2 Peters SAE, Huxley RR, Woodward M. Diabetes as a risk factor for stroke in women compared with men: a systematic review and metaanalysis of 64 cohorts, including 775385 individuals and 12539 strokes. The Lancet 2014;383:1973-80.

3 Gnatiuc L, Herrington WG, Halsey J, et al. Sex-specific relevance of diabetes to occlusive vascular and other mortality: a collaborative meta-analysis of individual data from 980793 adults from 68 prospective studies. Lancet Diabetes Endocrinol 2018;6:538-46.

4 Wannamethee SG, Papacosta O, Lawlor DA, et al. Do women exhibit greater differences in established and novel risk factors between diabetes and non-diabetes than men? The British Regional Heart Study and British Women's Heart Health Study. Diabetologia 2012;55:80-7.

5 Peters SAE, Huxley RR, Sattar N, et al. Sex differences in the excess risk of cardiovascular diseases associated with type 2 diabetes: 
potential explanations and clinical implications. Curr Cardiovasc Risk Rep 2015;9:36.

6 Juutilainen A, Kortelainen S, Lehto S, et al. Gender difference in the impact of type 2 diabetes on coronary heart disease risk. Diabetes Care 2004;27:2898-904.

7 Peters SAE, Huxley RR, Woodward M. Sex differences in body anthropometry and composition in individuals with and without diabetes in the UK Biobank. BMJ Open 2016;6:e010007.

8 Sattar N. Gender aspects in type 2 diabetes mellitus and cardiometabolic risk. Best Pract Res Clin Endocrinol Metab 2013;27:501-7.

9 Zhu S, St-Onge M-P, Heshka S, et al. Lifestyle behaviors associated with lower risk of having the metabolic syndrome. Metabolism 2004;53:1503-11.

10 Kautzky-Willer A, Harreiter J, Pacini G. Sex and gender differences in risk, pathophysiology and complications of type 2 diabetes mellitus. Endocr Rev 2016;37:278-316.

11 Kriska AM, Edelstein SL, Hamman RF, et al. Physical activity in individuals at risk for diabetes: diabetes prevention program. Med Sci Sports Exerc 2006;38:826-32.

12 Nelson KM, Reiber G, Boyko EJ, et al. Diet and exercise among adults with type 2 diabetes: findings from the third National health and nutrition examination survey (NHANES III). Diabetes Care 2002;25:1722-8.

13 Meisinger $\mathrm{C}$, Thorand B, Schneider A, et al. Lowel $\mathrm{H}$ : sex differences in risk factors for incident type 2 diabetes mellitus: the MONICA Augsburg cohort study. Arch Intern Med 2002;162:82-9.

14 Ladabaum U, Mannalithara A, Myer PA, et al. Obesity, abdominal obesity, physical activity, and caloric intake in US adults: 1988 to 2010. Am J Med 2014;127:717-27.

15 Perreault L, Ma Y, Dagogo-Jack S, et al. Sex differences in diabetes risk and the effect of intensive lifestyle modification in the diabetes prevention program. Diabetes Care 2008;31:1416-21.

16 Schram MT, Sep SJS, van der Kallen CJ, et al. The Maastricht study: an extensive phenotyping study on determinants of type 2 diabetes, its complications and its comorbidities. Eur J Epidemiol 2014;29:439-51.

17 Definition and diagnosis of diabetes mellitus and intermediate hyperglycemia: report of a WHO/IDF consultation. Geneva, Switzerland [article online] 2006.

18 Levy JC, Matthews DR, Hermans MP. Correct homeostasis model assessment (HOMA) evaluation uses the computer program. Diabetes Care 1998;21:2191-2.

19 Schram MT, Chaturvedi N, Schalkwijk CG, et al. Markers of inflammation are cross-sectionally associated with microvascular complications and cardiovascular disease in type 1 diabetes?the EURODIAB Prospective Complications Study. Diabetologia 2005;48:370-8.
20 van der Berg JD, Stehouwer CDA, Bosma H, et al. Koster A: dynamic sitting: measurement and associations with metabolic health. J Sports Sci 2019:1-9.

21 van Dongen MC, Wijckmans-Duysens NEG, den Biggelaar LJ, et al. The Maastricht FFQ: development and validation of a comprehensive food frequency questionnaire for the Maastricht study. Nutrition 2019;62:39-46.

22 Trichopoulou A, Orfanos P, Norat T, et al. Modified Mediterranean diet and survival: EPIC-elderly prospective cohort study. BMJ 2005;330.

23 JWR. Inleiding in de toegepaste biostatistiek 2007.

24 Donahue RP, Rejman K, Rafalson LB, et al. Sex differences in endothelial function markers before conversion to pre-diabetes: does the clock start ticking earlier among women?: the Western New York study. Diabetes Care 2007;30:354-9.

25 Haffner SM, Miettinen H, Stern MP. Relatively more atherogenic coronary heart disease risk factors in prediabetic women than in prediabetic men. Diabetologia 1997;40:711-7.

26 Du T, Fernandez C, Barshop R, et al. Sex differences in cardiovascular risk profile from childhood to midlife between individuals who did and did not develop diabetes at follow-up: the Bogalusa heart study. Diabetes Care 2019;42:635-43.

27 Logue J, Walker JJ, Colhoun HM, et al. Do men develop type 2 diabetes at lower body mass indices than women? Diabetologia 2011;54:3003-6.

28 Paul S, Thomas G, Majeed A, et al. Women develop type 2 diabetes at a higher body mass index than men. Diabetologia 2012;55:1556-7.

29 Peters SAE, Woodward M. Sex differences in the burden and complications of diabetes. Curr Diab Rep 2018;18:33.

30 Regensteiner JG, Golden S, Huebschmann AG, et al. Sex differences in the cardiovascular consequences of diabetes mellitus: a scientific statement from the American heart association. Circulation 2015:132:2424-47.

31 Woodward M, Peters SAE, Huxley RR. Diabetes and the female disadvantage. Womens Health 2015;11:833-9.

32 Bertram MY, Vos T. Quantifying the duration of pre-diabetes. Aust N Z J Public Health 2010;34:311-4.

33 Sandberg K, Ji H. Sex differences in primary hypertension. Biol Sex Differ 2012;3:7.

34 Kramer CK, Leitão CB, Canani LH, et al. Impact of white-coat hypertension on microvascular complications in type 2 diabetes. Diabetes Care 2008;31:2233-7.

35 Blokstra AVP, Venmans L, Holleman P. Nederland de Maat Genomen, 2009-2010. monitoring van risicofactoren in de algemenen bevolking. RIVM-rapport NR. 260152001/2011 Bilthoven, 2011. 